\title{
Dor lombar em adolescentes do semiárido: resultados de um censo populacional no município de Caracol (PI), Brasil
}

\author{
Low back pain among adolescents in the semiarid region: results \\ of a population census in the city of Caracol, State of Piauí, Brazil
}

Rodrigo Dalke Meucci ${ }^{1}$

Angélica Ozório Linhares ${ }^{2}$

Daniel Wenceslau Votto Olmedo ${ }^{1}$

Ewerton Luiz Porto Cousin Sobrinho ${ }^{1}$

Vlanice Madruga Duarte ${ }^{1}$

Juraci Almeida Cesar ${ }^{1}$

${ }^{1}$ Programa de PósGraduação em Saúde Pública, Universidade Federal do Rio Grande (FURG). R. Gen. Osório 102/4 ${ }^{\circ}$, Centro. $96203-900$ Rio Grande RS Brasil. rodrigodalke@gmail.com ${ }^{2}$ Programa de PósGraduação em Ciências da Saúde, FURG. Rio Grande RS Brasil.

\begin{abstract}
The scope of this article is to assess low back pain prevalence and associated factors in the last 12 months among adolescents in a city in the south of the State of Piaui. It was a cross-sectional study which included 1,112 adolescents aged 13-19 in the city of Caracol, Piaui. Demographic, socio-economic, nutritional, behavioral and comorbidty characteristics were investigated. The outcome was the occurrence of low back pain (LBP) in the last 12 months. The outcome prevalence was calculated in accordance with independent variables. The proportion of individuals with chronic LBP was calculated among adolescents with LBP in the last 12 months. Crude and adjusted Poisson regression was used to estimate the prevalence ratio in the analysis of associated factors. The prevalence of LBP in the last 12 months was $32.9 \%$. According to the adjusted analysis, being female $(P R=1.75$; 95\%CI:1.45-2.10), being aged 18-19 (PR = 1.27; 95\%CI:1.01-1.62), having higher income $(P R=1.33$; 95\%CI:1.061.67), having a job (PR 1.25 95\%CI:1.02-1.53) and having minor psychiatric disorders ( $P R 1.51$ 95\%CI: 1.25-1.82) were associated with LBP in the last 12 months. This study found a high prevalence of LBP among adolescents in a poor city in the semiarid region of Northeast Brazil.

Key words Low back pain, Back pain, Chronic low back pain, Adolescence, Epidemiology, Prevalence
\end{abstract}

Resumo O objetivo deste artigo é avaliar a prevalência e os fatores associados à dor lombar nos últimos 12 meses entre adolescentes de um município do sul do estado do Piauí, Brasil. Estudo transversal com 1112 adolescentes de 13-19 anos do municipio de Caracol. Investigaram-se características demográficas, socioeconômicas, nutricionais, comportamentais e comorbidades. $O$ desfecho foi a ocorrência de dor lombar (DL) nos últimos 12 meses. Foi calculada a prevalência do desfecho de acordo com as variáveis independentes. Entre os adolescentes com DL no último ano, foi calculada a proporção de indivíduos com dor lombar crônica. Para análise dos fatores associados, foi utilizada a regressão de Poisson bruta $e$ ajustada para cálculo das razões de prevalência. A prevalência de dor lombar nos últimos 12 meses foi de 32,9\%. De acordo com a análise ajustada, sexo feminino $(R P=1,75$; IC95\%:1,45-2,10), idade entre 18-19 anos ( $R P=1,27$; IC95\%:1,01-1,62), maior renda $(R P=1,33$; IC95\%:1,06-1,67), estar trabalhando (RP 1,25 IC95\%:1,02-1,53) e apresentar distúrbios psiquiátricos menores ( $R P$ 1,51 IC95\%: 1,25-1,82) permaneceram associados à DL nos últimos 12 meses. Este estudo encontrou elevada prevalência de DL entre adolescentes de um município pequeno do semiárido nordestino. Palavras-chave Dor lombar, Dor Lombar Crônica, Adolescência, Epidemiologia, Prevalência 


\section{Introdução}

A dor lombar (DL) é caracterizada como dor ou desconforto abaixo da décima segunda costela e acima da prega glútea inferior ${ }^{1}$. A DL é altamente incapacitante, sendo a principal causa global de anos vividos com incapacidade ${ }^{2}$. Trata-se de uma queixa comum e na maioria das vezes inespecífica, que acomete cerca de $80 \%$ da população adulta em algum momento da vida ${ }^{3}$.

De acordo com a literatura, a prevalência de DL em adolescentes varia não apenas devido a características das populações avaliadas, mas de acordo com a definição do desfecho, a qual possui grande heterogeneidade ${ }^{4}$. Um estudo de meta-análise estimou prevalências de 12,0\%,18,3\%, $33,6 \%$ e $39,9 \%$ para DL pontual, no último mês, nos últimos 12 meses e na vida, respectivamen$\mathrm{te}^{5}$. No Brasil, são poucos os estudos que avaliam a prevalência de DL em adolescentes. Estimativas de Porto Alegre, Bauru e Uruguaiana mostram que as prevalências de DL no último mês, no último ano e na vida, foram respectivamente de $31,6 \%, 19,5 \%$ e $16,1 \% \%^{6-8}$. Contrariamente ao estimado em outros países ${ }^{5}$, a prevalência de DL no último mês foi maior do que a encontrada para outros períodos de recordatório. Isso pode ser devido ao fato de que esta estimativa é proveniente de um estudo realizado com uma amostra não representativa da população alvo ${ }^{8}$. Além disso, evidencia a necessidade de mais estudos brasileiros sobre a prevalência de DL, de forma a melhorar a consistência dos achados.

Em relação aos fatores associados à DL entre adolescentes, pertencer ao sexo feminino $o^{6,7}$, possuir maior idade ${ }^{7}$, sobrepeso/obesidade ${ }^{7}$, permanecer número excessivo de horas assistindo televisão $^{6}$ e sintomas depressivos ${ }^{9}$ são fatores de risco para $\mathrm{DL}^{6,7}$. Entretanto, existem controvérsias na literatura, uma vez que outros estudos não encontraram associação entre DL e idade ${ }^{9,10}$, tempo assistindo televisã $0^{9,10}$, atividade física ${ }^{9,10}$ e escore de saúde mental ${ }^{10}$. A possibilidade de progressão da DL para a vida adulta, juntamente com seu potencial incapacitante, coloca como necessária a realização de estudos que meçam sua ocorrência e seus fatores associados, sobretudo em municípios de pequeno porte, baixo Índice de Desenvolvimento Humano Municipal (IDHM) e baixa renda per capita. Estas informações podem ser úteis ao planejamento dos serviços de saúde quanto à prevenção e o manejo da DL em adolescentes. Dessa forma, este estudo tem como objetivo avaliar a prevalência e os fatores associados à dor lombar no último ano em adolescentes de
Caracol, município pequeno e com baixo IDHM do sul do estado do Piauí.

\section{Métodos}

Estudo transversal de base populacional realizado no município de Caracol, localizado no sul do estado do Piauí, a cerca de $500 \mathrm{~km}$ de Teresina. Em 2010, Caracol tinha 10.212 habitantes e seu IDHM era de $0,55^{11}$. Foi realizado um censo de todos os adolescentes do município, sendo que dos 1133 indivíduos com idade entre 13 a 19 anos elegíveis para este estudo, foram entrevistados 1112 , o que corresponde a $1,9 \%$ de perdas e recusas. Foram considerados inelegíveis os indivíduos com limitação cognitiva ou física que impossibilitasse responder ao questionário, bem como aqueles que estavam institucionalizados.

Por se tratar de um censo populacional não foi realizado cálculo de tamanho amostral para o estudo da prevalência de DL nesta população. Entretanto, para exame dos fatores associados ao desfecho foi realizado cálculo de poder estatístico a posteriori, considerando os seguintes parâmetros: nível de confiança de 95\%; prevalência de DL entre não expostos de 20\%; razão exposto/ não exposto variando entre 1:1 a 1:9; e razão de prevalência de 1,6. De acordo com estes parâmetros, o número total de entrevistados garante um poder estatístico mínimo de $80 \%$ para detectar possíveis associações.

A coleta de dados foi realizada nos domicílios dos adolescentes, sendo executada por 8 entrevistadores e antropometristas treinados que eram estudantes de graduação do Campus de São Raimundo Nonato da Universidade Estadual do Piauí (UESPI). Utilizou-se um questionário que avaliou questões socioeconômicas (elaboradas pelos autores), demográficas, comportamentais e de dor nas costas (cervical, torácica e lombar), bem como o questionário autoaplicável Self Report Questionnaire (SRQ-20) ${ }^{12}$, que avaliou a presença de distúrbios psiquiátricos menores (DPM). Para mensuração de peso e altura foram utilizadas balança com precisão de $100 \mathrm{~g}$ e um antropômetro com precisão de $1 \mathrm{~cm}$. Todos os questionários foram revisados e codificados para serem duplamente digitados no programa Epi-Info 6.0. Cerca de 5\% dos indivíduos foram reentrevistados para controle de qualidade.

A DL foi caracterizada através da aplicação de uma versão modificada do questionário Nórdico $^{13}$. Os adolescentes que afirmaram ter tido dor nas costas no último ano eram orientados a 
apontar a localização exata da dor em uma figura humana de costas com as regiões cervical, torácica e lombar da coluna vertebral, cada uma delas identificadas por uma cor ${ }^{14}$. Os adolescentes que apontaram para a região lombar, área indicada na cor vermelha, foram considerados como tendo dor lombar. Entre aqueles que referiram DL, foi caracterizada a presença de dor lombar crônica a partir de resposta afirmativa quanto à presença de DL contínua por três meses ou mais no último ano. Além disso, foram investigadas a procura por atendimento médico e a falta ao trabalho/aula devido à DL.

Como variáveis independentes, foram avaliadas características sociodemográficas (sexo, idade, estado civil, renda familiar, área de residência e trabalho), antropométricas (Índice de Massa Corporal - IMC), comportamentais (tempo assistindo televisão e níveis de atividade física durante a semana) e comorbidades (distúrbios psiquiátricos menores), considerando como ponto de corte para resultado positivo 6 ou mais respostas positivas para o sexo masculino e 8 ou mais para o sexo feminino ${ }^{12}$. A renda per capita do domicílio ( $\mathrm{R} \$$ ) foi categorizada em tercis. O IMC foi categorizado em magreza, eutrofia e excesso de peso de acordo com a classificação proposta pela Organização Mundial da Saúde (OMS) ${ }^{15}$. A variável tempo assistindo televisão, coletada a partir da pergunta, "Quantas horas por dia você assiste televisão?", foi dicotomizada em até 2 horas e 2 horas ou mais diárias. Atividade física, coletada através da pergunta "Nos últimos sete dias, isto é, < desde dia da semana passada > até ontem, em quantos dias você praticou atividades físicas com pelo menos 1 hora de duração?", foi categorizada em inativos (0 dias), insuficientemente ativos (1-4 dias) e ativos (5-7 dias) ${ }^{16}$.

Os dados foram analisados no programa estatístico STATA 13 IC. Foi realizada uma análise descritiva dos adolescentes através do cálculo das proporções de cada uma das categorias das variáveis de interesse. Em seguida, foi calculada a prevalência do desfecho de acordo com as variáveis independentes. Dentre os adolescentes com DL no último ano, foram calculadas as proporções de indivíduos com dor lombar crônica, que faltaram à escola/trabalho e que procuraram atendimento médico.

Para análise dos fatores associados, foi utilizada a regressão de Poisson bruta e ajustada com variância robusta para cálculo das razões de prevalência e dos respectivos intervalos de confiança de 95\% (IC95\%). Foram consideradas associadas ao desfecho as variáveis com valor $\mathrm{p}<5 \%$ nos tes- tes de Wald de heterogeneidade ou de tendência linear para exposições ordinais. A medida de efeito utilizada foi a razão de prevalências (RP).

A análise ajustada com seleção para trás das variáveis foi realizada de acordo com um modelo hierárquico ${ }^{17}$ composto por três níveis: no primeiro, foram inseridas variáveis sociodemográficas (sexo, idade, renda, estado civil, área de residência e trabalho); no segundo, variáveis biológicas e comportamentais (IMC, atividade física, tempo assistindo televisão); e no terceiro, a comorbidade (distúrbios psiquiátricos menores). Foram mantidas no modelo as variáveis que apresentaram valor $\mathrm{p}<0,20$ dentro do seu nível, controlando para fatores de confusão do mesmo nível ou de níveis anteriores.

Este estudo foi aprovado pelo Comitê de Ética em Pesquisa da Faculdade de Medicina da Universidade Federal de Pelotas (CEPAS/UFPEL). Os adolescentes menores de 18 anos tiveram o termo de consentimento livre e esclarecido assinado pelos responsáveis. Os jovens menores de 18 anos tiveram seus termos assinados pelos responsáveis.

\section{Resultados}

De acordo com a Tabela 1, 53,3\% dos adolescentes eram do sexo feminino, 47,5\% tinham idade entre 15 e 17 anos, e 33,2\% pertenciam ao segundo tercil de renda familiar. Mais da metade dos indivíduos (57,5\%) viviam na área rural e 20\% tinham algum trabalho. Quanto ao estado nutricional, $84 \%$ dos adolescentes foram considerados eutróficos e 11\% tinham excesso de peso. Aproximadamente $75 \%$ dos adolescentes ficavam mais de 2 horas por dia assistindo TV, mais da metade $(54,6 \%)$ não praticava atividade física por mais de 60 minutos em nenhum dia da semana, e 23,0\% apresentavam distúrbios psiquiátricos menores. A prevalência geral de DL nos últimos 12 meses foi de 32,9\% (IC95\%: 30,1-35,7).

Dentre os adolescentes que relataram DL no último ano, 10,7\% tinham dor lombar crônica, $17,8 \%$ tinham faltado ao trabalho ou aula devido à DL e 22,7\% procuraram atendimento médico por causa dessa dor.

Indivíduos do sexo feminino, com idade entre 18-19 anos, maior renda, que viviam junto com companheiro, insuficientemente ativos e com distúrbios psiquiátricos menores apresentaram maiores prevalências do desfecho em relação aos seus grupos de comparação (Tabela 2).

De acordo com a análise ajustada, sexo feminino (RP 1,75 IC95\%: 1,45-2,10), idade entre 
Tabela 1. Descrição da amostra de adolescentes de 13 a 19 anos. Caracol, PI, 2011. ( $\mathrm{n}=1.112)$.

\begin{tabular}{|c|c|c|}
\hline Variável & $\mathbf{N}$ & $\%$ \\
\hline \multicolumn{3}{|l|}{ Sexo $(n=1.112)$} \\
\hline Masculino & 519 & 46,7 \\
\hline Feminino & 593 & 53,3 \\
\hline \multicolumn{3}{|l|}{ Idade $(\operatorname{anos})(\mathrm{n}=1.112)$} \\
\hline $13-14$ & 341 & 30,7 \\
\hline $15-17$ & 529 & 47,5 \\
\hline $18-19$ & 242 & 21,8 \\
\hline \multicolumn{3}{|l|}{ Vive junto com companheiro $(\mathrm{n}=1.112)$} \\
\hline Não & 1003 & 90,2 \\
\hline $\operatorname{Sim}$ & 109 & 9,8 \\
\hline \multicolumn{3}{|l|}{ Renda familiar (em reais) $(\mathrm{n}=1.112)$} \\
\hline Tercil mais pobre & 375 & 33,7 \\
\hline Tercil intermediário & 369 & 33,2 \\
\hline Tercil mais rico & 368 & 33,1 \\
\hline \multicolumn{3}{|l|}{ Área de residência $(\mathrm{n}=1.112)$} \\
\hline Urbana & 472 & 42,5 \\
\hline Rural & 640 & 57,5 \\
\hline \multicolumn{3}{|l|}{ Está trabalhando $(\mathrm{n}=1.112)$} \\
\hline Não & 890 & 80,0 \\
\hline Sim & 222 & 20,0 \\
\hline \multicolumn{3}{|l|}{ Estado nutricional (OMS) $(\mathrm{n}=1.111)$} \\
\hline Magro & 55 & 5,0 \\
\hline Eutrofia & 934 & 84,0 \\
\hline Excesso de peso & 122 & 11,0 \\
\hline \multicolumn{3}{|l|}{ Tempo na frente da TV $(\mathrm{n}=1.112)$} \\
\hline Até 2 horas & 268 & 24,1 \\
\hline 2 horas ou mais & 844 & 75,9 \\
\hline \multicolumn{3}{|c|}{ Atividade física (dias que realizou mais que 60 min na última semana) $(\mathrm{n}=1.112$ ) } \\
\hline Inativos (0 dias) & 607 & 54,6 \\
\hline Insuficientemente ativos $(1-4$ dias $)$ & 275 & 24,7 \\
\hline Ativos $(5-7$ dias $)$ & 230 & 20,7 \\
\hline \multicolumn{3}{|l|}{ Prevalência geral de DPM $(\mathrm{n}=906)$} \\
\hline Negativo & 698 & 77,0 \\
\hline Positivo & 209 & 23,0 \\
\hline \multicolumn{3}{|l|}{ Dor lombar $(\mathrm{n}=1.109)$} \\
\hline Não & 744 & 67,1 \\
\hline Sim & 365 & 32,9 \\
\hline
\end{tabular}

DPM - Distúrbios psiquiátricos menores. DPM - Minor psychiatric disorders.

18-19 anos (RP 1,27 IC95\%: 1,01-1,62), maior renda (RP 1,33 IC95\%: 1,06-1,67), estar trabalhando (RP 1,25 IC95\%: 1,02-1,53) e apresentar distúrbios psiquiátricos menores ( $\mathrm{RP} 1,51$ IC95\%: 1,25-1,82) permaneceram associados ao desfecho (Tabela 3). Não foram associadas as variáveis IMC, nível de atividade física e tempo assistindo TV.

\section{Discussão}

No presente estudo, a prevalência do desfecho foi de $32,9 \%$, sendo que $11 \%$ dos adolescentes que relataram DL nos últimos doze meses tinham dor lombar crônica e um quinto havia procurado por atendimento médico. Pertencer ao sexo feminino, possuir maior idade e renda, estar trabalhando e positividade para distúrbios psiquiátricos foram 
Tabela 2. Prevalência da dor lombar no último ano em adolescentes. Caracol, PI, 2011. ( $\mathrm{n}=1.112)$.

\begin{tabular}{|c|c|c|c|}
\hline \multirow{2}{*}{ Variável } & \multicolumn{2}{|c|}{ Dor Lombar } & \multirow{2}{*}{ valor-p ${ }^{\star}$} \\
\hline & $\mathbf{P}(\%)$ & RP (IC 95\%) & \\
\hline $\operatorname{Sexo}(n=1.112)$ & & & $<0,001$ \\
\hline Masculino & 24,6 & 1,00 & \\
\hline Feminino & 40,2 & $1,64(1,37-1,96)$ & \\
\hline Idade $(\operatorname{anos})(\mathrm{n}=1.112)$ & & & $0,001^{* *}$ \\
\hline $13-14$ & 27,1 & 1,0 & \\
\hline $15-17$ & 34,7 & $1,28(1,04-1,58)$ & \\
\hline $18-19$ & 37,2 & $1,37(1,08-1,75)$ & \\
\hline Vive junto com companheiro $(\mathrm{n}=1.112)$ & & & 0,03 \\
\hline Não & 32,0 & 1,0 & \\
\hline Sim & 41,7 & $1,30(1,02-1,66)$ & \\
\hline Renda familiar (em reais) $(\mathrm{n}=1.112)$ & & & $0,06^{\star *}$ \\
\hline Tercil mais pobre & 28,7 & 1,0 & \\
\hline Tercil intermediário & 35,0 & $1,22(0,99-1,51)$ & \\
\hline Tercil mais rico & 35,2 & $1,23(0,99-1,51)$ & \\
\hline Área de residência $(\mathrm{n}=1.112)$ & & & 0,36 \\
\hline Urbana & 31,4 & 1,0 & \\
\hline Rural & 34,0 & $1,08(0,91-1,29)$ & \\
\hline Está trabalhando $(\mathrm{n}=1.112)$ & & & 0,10 \\
\hline Não & 31,8 & 1,0 & \\
\hline Sim & 37,4 & $1,18(0,97-1,43)$ & \\
\hline Estado nutricional (OMS) $(\mathrm{n}=1.111)$ & & & 0,45 \\
\hline Magreza & 30,1 & 1,0 & \\
\hline Eutrofia & 32,3 & $1,05(0,70-1,57)$ & \\
\hline Excesso de peso & 37,7 & $1,22(0,77-1,93)$ & \\
\hline Tempo na frente na TV $(\mathrm{n}=1.112)$ & & & 0,79 \\
\hline Até 2 horas & 33,6 & 1,0 & \\
\hline 2 horas ou mais & 32,7 & $0,97(0,80-1,18)$ & \\
\hline $\begin{array}{l}\text { Atividade física (dias que realizou mais que } 60 \text { min na última } \\
\text { semana) }(\mathrm{n}=1.112)\end{array}$ & & & $0,001^{\star *}$ \\
\hline Inativos (0 dias) & 37,1 & $1,58(1,22-2,04)$ & \\
\hline Insuficientemente ativos ( $1-4$ dias $)$ & 31,5 & $1,34(1,01-1,80)$ & \\
\hline Ativos (5 - 7 dias) & 23,5 & 1,0 & \\
\hline Prevalência geral de DPM $(\mathrm{n}=906)$ & & & $<0,001$ \\
\hline Negativo & 28,4 & 1,0 & \\
\hline Positivo & 44,5 & $1,57(1,29-1,90)$ & \\
\hline
\end{tabular}

identificados como fatores significativamente associados ao desfecho.

A prevalência encontrada neste estudo é semelhante à relatada em outros países $(33,6 \%$; IC95\%: 26,9-41,0 $)^{5}$. Entretanto, no Brasil, um estudo de base escolar que utilizou o mesmo período de recordatório (DL nos últimos 12 meses) relatou prevalência menor de DL entre escolares de 11 a 14 anos da cidade de Bauru (19,5\%). Além das diferentes estruturas etárias dos indivíduos avaliados, no presente estudo, foram inclu- ídos adolescentes que não estavam frequentando a escola. Além disso, os municípios avaliados são completamente distintos em relação a características socioeconômicas, sendo que Caracol possuía, em 2010, um IDH-M baixo (0,55), enquanto Bauru tinha um IDH-M considerado muito alto $(0,80)^{18}$. Isso pode ter contribuído para a maior prevalência de DL entre os adolescentes de Caracol.

A procura de atendimento médico gerada pela dor lombar nos últimos 12 meses pode ser 
Tabela 3. Análise ajustada dos fatores associados à dor lombar no último ano em adolescentes. Caracol, PI, 2011. $(\mathrm{n}=1.112)$.

\begin{tabular}{|c|c|c|}
\hline \multirow{2}{*}{ Variável } & Dor Lombar & \multirow{2}{*}{ valor- $\mathrm{p}^{\star}$} \\
\hline & RP (IC 95\%) & \\
\hline Sexo $(n=1.112)$ & & $<0,001$ \\
\hline Masculino & 1,0 & \\
\hline Feminino & $1,75(1,45-2,10)$ & \\
\hline Idade $(\operatorname{anos})(\mathrm{n}=1.112)$ & & $0,04^{\star *}$ \\
\hline $13-14$ & 1,0 & \\
\hline $15-17$ & $1,23(1,00-1,52)$ & \\
\hline $18-19$ & $1,27(1,01-1,62)$ & \\
\hline Renda familiar (em reais) $(\mathrm{n}=1.112)$ & & $0,01^{\star *}$ \\
\hline Tercil mais pobre & 1,0 & \\
\hline Tercil intermediário & $1,27(1,02-1,57)$ & \\
\hline Tercil mais rico & $1,33(1,06-1,67)$ & \\
\hline Área de residência $(\mathrm{n}=1.112)$ & & 0,12 \\
\hline Urbana & 1,0 & \\
\hline Rural & $1,15(0,96-1,37)$ & \\
\hline Está trabalhando $(\mathrm{n}=1.112)$ & & 0,04 \\
\hline Não & 1,0 & \\
\hline Sim & $1,25(1,02-1,53)$ & \\
\hline $\begin{array}{l}\text { Atividade física (dias que realizou mais que } 60 \mathrm{~min} \text { na última } \\
\text { semana) }(\mathrm{n}=1.112)\end{array}$ & & $0,16^{* *}$ \\
\hline Inativos (0 dias) & $1,24(0,95-1,63)$ & \\
\hline Insuficientemente ativos ( $1-4$ dias) & $1,27(0,96-1,70)$ & \\
\hline Ativos (5 - 7 dias) & 1,0 & \\
\hline Prevalência geral de DPM ( $\mathrm{n}=906)$ & & $<0,001$ \\
\hline Negativo & 1,0 & \\
\hline Positivo & $1,51(1,25-1,82)$ & \\
\hline
\end{tabular}

considerada baixa, embora a comparabilidade seja limitada pela escassez de estudos que avaliaram a utilização de serviço por DL entre adolescentes. A falta ao trabalho/aula é um indicador de gravidade e este resultado é relatado em outro estudo $^{19}$. A prevalência de dor lombar crônica entre os adolescentes com DL pode ser considerada alta, o que é preocupante nesta etapa da vida, uma vez que sintomas crônicos são associados à incapacidade entre adultos ${ }^{3}$.

A associação entre sexo feminino e DL nos últimos 12 meses é consistente com a literatura, mesmo entre estudos que utilizam diferentes períodos de recordatório para caracterização do desfecho ${ }^{4,20}$. Estes resultados podem refletir diferenças estruturais e psicossociais entre os sexos, sendo que as mulheres podem relatar a dor com maior frequência do que os homens. Além disso, diferenças na estrutura músculo-esquelética po- dem tornar as mulheres mais predispostas à DL, uma vez que tenham maior gasto energético do que os homens quando expostas a demandas físicas similares ${ }^{6}$. Entretanto, as razões pelas quais as mulheres relatam mais DL do que os homens são especulativas ${ }^{6}$. Cabe ressaltar ainda, que neste estudo não foi avaliado se a DL entre as mulheres era decorrente da menstruação, o que pode ter superestimado a prevalência e, consequentemente, o risco de DL neste grupo em relação aos homens.

A maior sobrecarga sobre a coluna vertebral devido aos longos períodos sentados e o carregamento de peso que aumentam na medida em que os adolescentes se tornam mais velhos pode explicar a associação linear direta entre idade e o desfecho ${ }^{4,7,8,21}$.

Não foram encontrados estudos que avaliem a associação entre maior renda e DL no último 
ano, sendo que apenas um estudo brasileiro avaliou a relação entre nível socioeconômico e DL, mas não encontrou diferença significativa ${ }^{7}$. Por se tratar de um município com IDHM baixo, adolescentes que trabalham possuem maior renda, sendo desta forma, mais expostos a cargas de trabalho que podem predispor à DL. Estudo realizado em Pelotas, com crianças e adolescentes, observou que alguns tipos de trabalho estavam associados à dor nas costas, tais como trabalhos domésticos e trabalho em indústrias ${ }^{22}$. No entanto, esta comparação é limitada devido às diferentes definições dos desfechos e às diferenças socioeconômicas entre os municípios.

Dentre os estudos brasileiros, apenas um encontrou associação entre hiperatividade e sintomas emocionais com DL em adolescentes ${ }^{8}$. Entretanto, além de não ser um estudo de base populacional, o instrumento utilizado foi o questionário de capacidade e dificuldades - SDQ, o qual difere substancialmente do SRQ-20. É importante ressaltar que o delineamento utilizado limita a avaliação da temporalidade da associação encontrada, e que este achado é controverso na literatura. Enquanto alguns estudos relatam associação, outros não encontram resultados semelhantes ${ }^{9,10}$. Neste caso, o uso de diferentes instrumentos e definições para mensurar variáveis psicossociais pode contribuir para achados contraditórios.

Neste estudo, não houve associação significativa entre atividade física e DL, achado consistente com um estudo de coorte com adolescentes do ensino médio do Canadá ${ }^{10}$. Entretanto, além das diferenças metodológicas, bem como em relação à idade média dos participantes, neste estudo, não foram avaliadas em detalhes as atividades físicas praticadas pelos adolescentes, o que limita a capacidade de discernir entre as que seriam benéficas das que seriam de risco para DL. Desta forma, persistem muitas incertezas na literatura sobre quais atividades físicas seriam protetoras e quais seriam de risco para $\mathrm{DL}^{10,20}$.

Embora um estudo brasileiro apresente maior risco de DL entre indivíduos com sobrepeso/obesidade ${ }^{7}$, não foi encontrada associação estatisticamente significativa entre estado nutricional e DL. Um aspecto que pode ser mencionado é a baixa proporção de adolescentes com excesso de peso (11\%) no presente estudo, o que pode ter contribuído para este achado negativo.
Por se tratar de um estudo transversal, a interpretação dos achados em relação aos fatores associados à DL nos últimos 12 meses requer considerar que algumas das associações podem ser decorrentes de causalidade reversa. Este estudo também pode apresentar erro de recordatório pelo desfecho ter sido caracterizado como prevalência no último ano. Desta forma, a alta prevalência de DL nos últimos 12 meses pode ter sido subestimada. Assim, a proporção encontrada é o número mínimo de casos esperados na população avaliada.

O estudo da prevalência e dos fatores associados à DL em adolescentes pode ser uma ferramenta útil na busca por intervenções que visem o tratamento da DL. De acordo com uma revisão sistemática recente, existem evidências de que a realização de exercícios pode reduzir os níveis de dor em curto prazo $^{23}$. Dessa forma, é recomendável que profissionais de saúde procurem identificar adolescentes com DL de forma a tratar adequadamente estes indivíduos. Em relação a medidas preventivas, os achados na literatura são controversos e não há evidência de que intervenções ergonômicas ou educativas sejam eficazes na prevenção da DL entre adolescentes ${ }^{23,24}$. Neste sentido, a baixa qualidade metodológica contribui para que os achados da maioria dos estudos de intervenção sejam inválidos ${ }^{23}$.

Deste modo, existem importantes lacunas no conhecimento sobre a ocorrência e os fatores associados à DL em adolescentes, bem como em relação ao seu tratamento e prevenção. São necessários, desta forma, mais estudos observacionais e experimentais metodologicamente adequados sobre o tema.

\section{Conclusão}

Por ser um censo com adolescentes de um município com baixo IDHM do semiárido nordestino, este estudo traz uma contribuição original ao estudo da DL. Evidencia elevada prevalência de DL (32,9\%), bem como identifica os principais fatores associados (sexo feminino, possuir maior idade e renda, estar trabalhando e positividade para distúrbios psiquiátricos). Estes achados podem ser úteis a profissionais que trabalhem com adolescentes, de forma que identifiquem oportunamente os indivíduos mais suscetíveis. 


\section{Colaboradores}

RD Meucci, AO Linhares, DWV Olmedo e ELPC Sobrinho realizaram a revisão de literatura, a análise dos dados e elaboração do artigo. VM Duarte participou da coleta e revisão dos dados e da elaboração do artigo. JA Cesar elaborou o projeto de onde o estudo foi originado, supervisionou a coleta e entrada de dados e revisou o artigo final. Todos os autores contribuíram de maneira fundamental para a realização desse estudo e leram e aprovaram o conteúdo do manuscrito.

\section{Referências}

1. Andersson JAD. Problems of classification of low back pain. Rheumatol Rehabil 1977; 16:34-36.

2. Global-Burden-of-Disease-Study-2013-Collaborators. Global, regional, and national incidence, prevalence, and years lived with disability for 301 acute and chronic diseases and injuries in 188 countries, 1990-2013: a systematic analysis for the Global Burden of Disease Study 2013. Lancet 2015; 386(9995):743-800.

3. Diamond S, Borenstein D. Chronic low back pain in a working-age adult. Best Pract Res Clin Rheumatol 2006; 20(4):707-720.

4. Calvo-Munoz I, Gomez-Conesa A, Sanchez-Meca J. Prevalence of low back pain during childhood and adolescence: a systematic review. Rev Esp Salud Publica 2012; 86(4):331-353.

5. Calvo-Munoz I, Gomez-Conesa A, Sanchez-Meca J. Prevalence of low back pain in children and adolescents: a meta-analysis. BMC Pediatrics 2013; 13:14.

6. De-Vitta A, Martinez MG, Piza NT, Simeão SFdAP, Ferreira NP. Prevalência e fatores associados à dor lombar em escolares. Cad Saude Publica 2011; 27(8):15201528.

7. Graup S, Bergmann MLdA, Bergmann GG. Prevalência de dor lombar inespecífica e fatores associados em adolescentes de Uruguaiana/RS. Rev Bras Ortop 2014; 49(6):661-667.

8. Lemos ATd, Santos FRd, Moreira RB, Machado DT, Braga FCC, Gaya ACA. Ocorrência de dor lombar e fatores associados em crianças e adolescentes de uma escola privada do sul do Brasil. Cad Saude Publica 2013; 29(11):2177-2185.

9. Diepenmaat AC, van-der-Wal MF, de-Vet HC, Hirasing RA. Neck/shoulder, low back, and arm pain in relation to computer use, physical activity, stress, and depression among Dutch adolescents. Pediatrics 2006 117(2):412-416.

10. Feldman DE, Shrier I, Rossignol M, Abenhaim L. Risk factors for the development of low back pain in adolescence. Am J Epidemiol 2001; 154(1):30-36.

11. Instituto Brasileiro de Geografia e Estatística (IBGE). IBGE Cidades. 2016 [cited 2016 Sep 2]. Available from:

12. Mari JJ, Williams P. A validity study of a psychiatric screening questionnaire (SRQ-20) in primary care in the city of Sao Paulo. Br J Psychiatry 1986; 148(1):2326.

13. Kuorinka I, Jonsson B, Kilbom A, Vinterberg H, Biering-Sørensen F, Andersson G, et al. Standardised Nordic questionnaires for the analysis of musculoskeletal symptoms. Appl Ergon 1987; 18(3):233-237.
14. Meucci RD, Fassa AG, Paniz VM, Silva MC, Wegman $\mathrm{DH}$. Increase of chronic low back pain prevalence in a medium-sized city of southern Brazil. BMC Musculoskelet Disord 2013; 14(1):155.

15. Cole TJ, Flegal KM, Nicholls D, Jackson AA. Body mass index cut offs to define thinness in children and adolescents: international survey. BMJ 2007; 335(7612):166167.

16. United States Department of Health and Human Services (USDHHS). Physical Activity Guidelines Advisory Committee Report 2008. Washington: USDHHS; 2008.

17. Victora CG, Huttly SR, Fuchs SC, AOlinto MT. The role of conceptual frameworks in epidemiological analysis: a hierarchical approach. Int J Epidemiol 1997; 26(1):224-227.

18. Instituto de Pesquisa Econômica Aplicada (IPEA). Indice de Desenvolvimento Humano Municipal Brasileiro: Série Atlas do Desenvolvimento Humano no Brasil 2013. Brasília: IPEA; 2013.

19. Bejia I, Abid N, Ben-Salem K, Letaief M, Younes M, Touzi M, Bergaoui N. Low back pain in a cohort of 622 Tunisian schoolchildren and adolescents: an epidemiological study. Eur Spine J 2005; 14(4):331-336.

20. Balagué F, Troussier B, Salminen JJ. Non-specific low back pain in children and adolescents: risk factors. Eur Spine J 1999; 8(6):429-438.

21. Silva MR, Badaró AF. MMD-A. Low back pain in adolescent and associated factors: A cross sectional study with schoolchildren. Braz J Phys Ther 2014; 18(5):402409.

22. Fassa AG, Facchini LA, Dall'Agnol MM, Christiani DC. Child labor and musculoskeletal disorders: the Pelotas (Brazil) epidemiological survey. Public Health Rep 2005; 120(6):665-673.

23. Michaleff ZA, Kamper SJ, Maher CG, Evans R, Broderick C, Henschke N. Low back pain in children and adolescents: a systematic review and meta-analysis evaluating the effectiveness of conservative interventions. Eur Spine J 2014; 23(10):2046-2058.

24. Burton AK. How to prevent low back pain. Best Pract Res Clin Rheumatol 2005; 19(4):541-555.

Artigo apresentado em 16/11/2015

Aprovado em 18/05/2016

Versão final apresentada em 20/05/2016 